\title{
Properties and Photoactivity of Rhodopsin Mutants
}

\author{
THOMAS P. SAKMAR a,* AND KARIM FAHMY \\ 'The Howard Hughes Medical Institute, Laboratory of Molecular Biology and Biochemistry, Rockefeller University, \\ 1230 York Avenue, New York, New York 10021, USA \\ 'Institut für Biophysik und Strahlenbiologie, Albert-Lüdwigs-Universität, Albertstraße 23, 79104 Freiburg, Germany
}

(Received 6 May 1995 and in revised form 22 August 1995)

\begin{abstract}
A variety of spectroscopic and biochemical studies of recombinant site-directed mutants of rhodopsin and related visual pigments have been carried out. These studies have elucidated key structural elements common to visual pigments, such as a conserved disulfide bond. In addition, systematic analysis of the chromophore-binding pocket in rhodopsin and cone pigments has led to an improved understanding of the mechanism of the opsin-shift, and of particular molecular determinants underlying color vision in humans. Identification of conformational changes which occur upon rhodopsin photoactivation has been of particular recent concern. Assignment of these molecular alterations to specific regions in the receptor has been attempted by studying native opsin regenerated with synthetic retinal analogues or recombinant opsins regenerated with 11-cis-retinal. Individual molecular groups that undergo structural alterations during photoactivation have been identified. Analysis of particular mutant pigments in which specific groups are locked into their respective "on" or "off" states has provided a framework to identify determinants of the active conformation as well as the minimal number of intramolecular transitions required to switch between inactive and active conformations. A simple model for the active state of rhodopsin can be compared to structural models of its ground state to localize chromophore-protein interactions that may be important in the photoactivation mechanism.
\end{abstract}

\section{INTRODUCTION}

Visual pigments comprise a branch of a large family of G-protein-coupled receptors. Although they share many similarities with other G-protein-coupled receptor types, there is significant specialization in visual pigments not found in other receptor families. In particular, pigments are made up of opsin apoprotein plus chromophore. The chromophore is a cofactor and not a ligand as in the other seven helix receptors. It is linked covalently via a Schiff base bond to a specific lysine residue. The chromophore-binding pocket resides in the membrane-embedded domain of the protein. One of two retinoids serves as the chromophore for all visual pigments. The chromophore in most vertebrate pigments is the aldehyde of vitamin A, 11-cis-retinal. The chromophore in invertebrates and in some fish and amphibians is the aldehyde of vitamin $A_{2}, 11$-cis-3-dehydroretinal, which contains an additional carbon-carbon double bond in the $\beta$-ionone ring.

Photoisomerization of the 11-cis to all-trans form of the retinylidene chromophore is the primary event in visual signal transduction, and it is the only light-dependent step. Chromophore isomerization activates the pigment, allowing it to interact with a specific heterotrimertic $\mathrm{G}$ protein. In the case of the vertebrate visual system, G-protein activation leads to the activation of a cyclic-GMP phosphodiesterase, and the closing of a cyclic-GMP-gated cation channel. In short, light causes a graded hyperpolarization of the photoreceptor cell. The amplification, modulation, and regulation of the light response is of great physiological importance and has been discussed in detail elsewhere. ${ }^{1}$

The visual pigments of many species of vertebrates and invertebrates have been studied by absorption spectroscopy or microspectrophotometry of visual organs. Therefore, historically, vertebrate visual pigments have been classified generally based on the photoreceptor cell type of the retina in which they are found. ${ }^{2}$ Rod cells, responsible for dim-light vision, contain rhodopsin

*Author to whom correspondence should be addressed.

$$
\text { Israel Journal of Chemistry } \quad \text { Vol. } 35 \quad 1995 \quad \text { pp. 325-337 }
$$


("red" opsin). Cone cells, responsible for bright-light and color vision, contain iodoopsins ("violet" opsin), also known as cone pigments or color vision pigments. The cloning of opsins from a variety of species has allowed more detailed comparisons and phylogenetic classifications based on structural, spectral, and biochemical properties of visual pigments. ${ }^{3}$ The homology in the opsin family of genes indicates that divergent evolution occurred from a single precursor retinal-binding protein to form long- and short-wavelength-absorbing prototypes. The long wavelength prototype diverged to form red and green pigments. The short wavelength prototype then diverged to form a blue pigment and the family of rhodopsins and rhodopsin-like green pigments.

Each visual pigment is tuned to a particular wavelength of maximal absorption, $\lambda_{\max }$, although the visible absorbance peak tends to be quite broad. Even though retinal is the universal chromophore, the $\lambda_{\max }$ values of visual pigments span the visible spectrum, i.e., from near ultraviolet at about $400 \mathrm{~nm}$ to far visible red at about $600 \mathrm{~nm}$. Distinct chromophore-protein interactions are responsible directly or indirectly for spectral tuning in visual pigments. Thus, differences in primary structure result in differences in spectral properties.

A number of structural features are shared by visual pigments. Like all G-protein-coupled receptors, they consist of seven hydrophobic domains. A Glu or Asp/ $\mathrm{Arg} / \mathrm{Tyr}$ or Phe tripeptide sequence is found at the cytoplasmic border of the third transmembrane domain. This domain is conserved in most G-protein-coupled receptors and has been shown to be involved in G-protein interaction. A lysine residue that acts as the linkage site for the chromophore is conserved within the seventh transmembrane segment in all pigments. A pair of highly conserved cysteine residues is found on the extracelluar surface and forms a disulfide bond. In many pigments, a carboxylic acid residue that acts as the counterion to the protonated, positively-charged Schiff base is conserved within the third transmembrane segment. Sites of light-dependent phosphorylation (serine and threonine residues) are found at the carboxyl-terminal tail of most visual pigments. These sites may be analogous to phosphorylation sites found on the carboxyl-terminal tails of other G-protein-coupled receptors. ${ }^{4}$

Bovine rhodopsin is the most extensively studied Gprotein-coupled receptor. A large amount of pigment can be obtained from a single bovine retina by sucrose density gradient centrifugation preparation of the rod outer segment disc membranes. The pigment can be purified further by lectin affinity chromatography on concanavalin A Sepharose resin. Rhodopsin is stable in solubilized form in a variety of detergents, including digitonin, dodecylmaltoside, and octyl glucoside. Bovine rhodopsin was the first G-protein-coupled receptor to be sequenced by amino acid sequencing ${ }^{5}$ and the first to be cloned. ${ }^{6}$ The cloning of a $\beta$-adrenergic receptor ${ }^{7}$ led to the identification of the structural homologies that now define the large family of G-protein-coupled receptors.

Bovine rhodopsin is 348 amino acid residues in length, and is identical to human rhodopsin at all but 23 positions. ${ }^{6}$ Hydrophobicity profiles are consistent with seven transmembrane segments (Fig. 1). The amino-

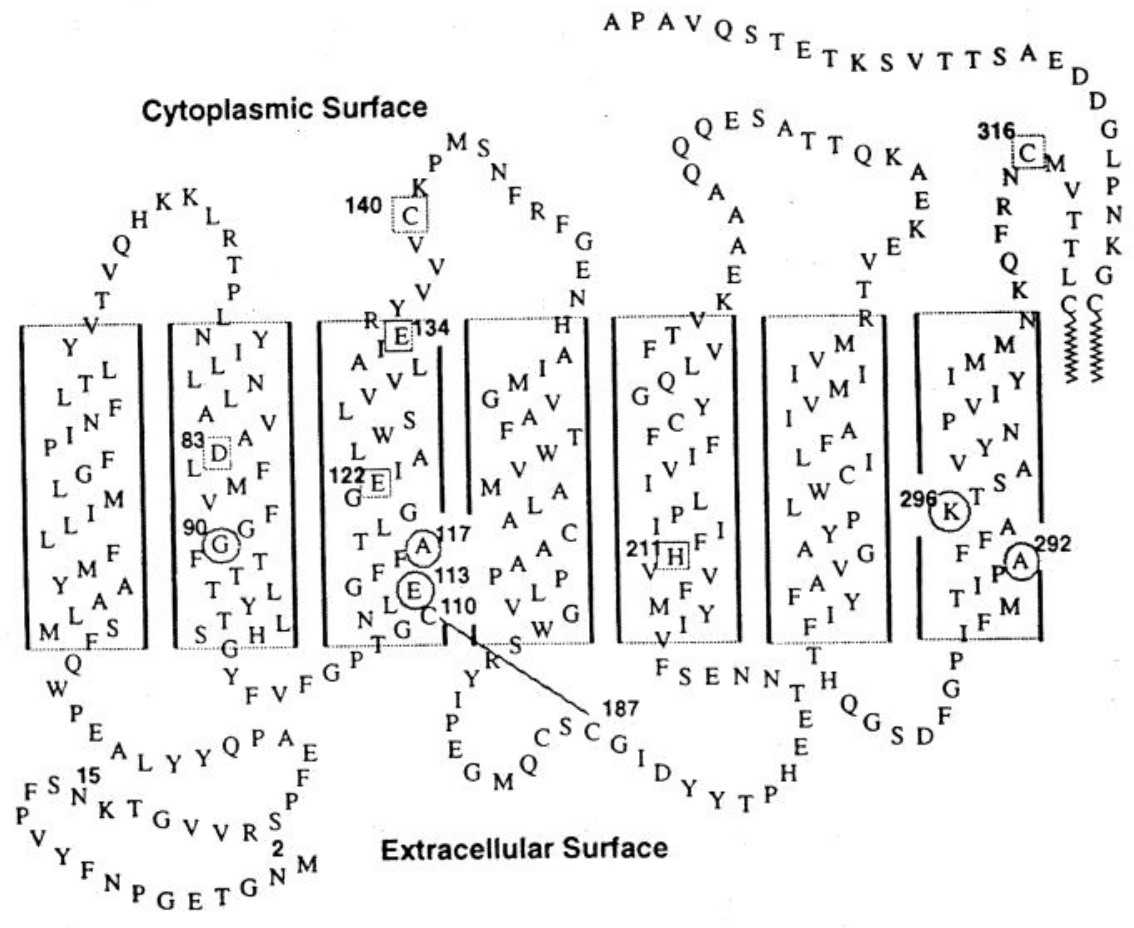

Fig. 1. Schematic representation of bovine rhodopsin secondary structure. Seven putative transmembrane helices based on previous models are shown. The carboxyl terminus and cytoplasmic surface is toward the top, and the amino terminus and extracellular (intradiscal) surface is toward the bottom of the figure. Amino acid positions discussed in the text are numbered. Lys-296 is the site of the retinylidene Schiff base linkage. Glu-113 is the negatively charged counterion to the positively charged protonated Schiff base. Other significant residues that have been mutated in studies of the Schiff base environment are also circled: Gly-90, Ala-117, Ala-292. 
terminal domain is remarkable for two N-linked glycosylation sites. The carboxyl-terminal domain is rich in serine and threonine residues, which are phosphorylated in a light-dependent manner by rhodopsin kinase. The retinal Schiff base linkage is at Lys-296. Rhodopsin has a broad absorption maximum $\left(\lambda_{\max }\right)$ at about $500 \mathrm{~nm}$. Upon photoisomerization of the chromophore, the pigment is converted to metarhodopsin II (MII) with a $\lambda_{\max }$ value of $380 \mathrm{~nm}$. The MII intermediate is characterized by a deprotonated Schiff base chromophore linkage. MII is the active form of the receptor $\left(\mathrm{R}^{*}\right)$, which catalyzes guanine-nucleotide exchange by the rod cell $G$ protein, transducin.

A number of studies have been carried out on bovine rhodopsin to investigate the retinal-binding domain of bovine rhodopsin. Several spectroscopic methods such as resonance Raman spectroscopy, Fourier-transform infrared difference spectroscopy, and NMR spectroscopy have been employed. ${ }^{8.9}$ Other approaches have included reconstitution of opsin apoprotein with synthetic retinal analogues ${ }^{10}$ and photochemical crosslinking. ${ }^{11.12}$ Early work on the structure and function of recombinant bovine rhodopsin focusing on the use of techniques of molecular biology has been reviewed. ${ }^{13}$

A key advance in the molecular genetics of human hereditary blinding diseases has led to considerable work involving recombinant rhodopsins. Retinitis pigmentosa is a group of hereditary progressive blinding diseases with variable clinical presentations. One form of the disease, autosomal dominant retinitis pigmentosa (ADRP), was linked to a mutation in the gene for rhodopsin. ${ }^{14}$ This mutation at codon 23 would result in the change of a proline residue to a histidine residue. Over 40 other mutations have been reported in ADRP patients. ${ }^{14,15}$ Several studies have been carried out in which site-directed mutant opsin genes corresponding to ADRP genotypes were prepared ${ }^{16}$ When expressed in tissue culture, the mutant opsins display a heterogeneity of spectral properties, biochemical properties, and cellular transport behavior. Some mutants are defective in chromophore binding, others in cellular transport and insertion into the plasma membrane. However, some mutations have no apparent effect. One mutation linked to ADRP is a replacement of the Schiff base lysine by glutamic acid. This mutation should prevent chromophore Schiff base formation. Interestingly, a similar mutant of bovine rhodopsin was shown to have constitutive activity without chromophore in in-vitro transducin-activation assays. ${ }^{17}$ Significant work in this area is continuing using transgenic mouse technology to produce mouse models of retinal degeneration.

Recently, site-directed mutagenesis in combination with a variety of spectroscopic and biochemical techniques has provided useful information about the retinal-binding pocket and the molecular mechanism of rhodopsin photoactivation. This review focuses on the recent functional characterization of site-directed mutants of bovine rhodopsin and some cone pigments. In addition, an attempt is made to reconcile previous key findings and existing structural models with information gained from the analysis of site-directed mutant pigments.

\section{THE STRUCTURAL ROLE OF INTRADISCAL DOMAIN AMINO ACID RESIDUES}

The topology of rhodopsin with respect to the disc membrane bilayer is defined by three structural domains; the extracellular (intradiscal) domain, the membrane-embedded domain, and the intracellular (cytoplasmic) domain. The role of the extracellular domain in rhodopsin structure and function has been elucidated by a number of studies involving site-directed mutagenesis. This work has potential relevance in understanding the molecular pathophysiology of some forms of ADRP which are due to missense mutations in the portions of the rhodopsin gene encoding this domain. ${ }^{14-16}$ Transgenic mice expressing the $\mathrm{P} 23 \mathrm{H}$ mutation have been characterized, and results indicate that retinal degeneration may be due to improper mutant opsin expression and cellular transport. ${ }^{18}$ Further work is need to clarify how these defects lead to photoreceptor cell death.

The extracellular loops and amino-terminal tail of bovine rhodopsin have been shown in a deletion analysis to be important for proper folding of the receptor that allows cellular processing and chromophore binding. ${ }^{19}$ Insertional mutagenesis was also used in a related study to probe the topology of rhodopsin and to correlate the location of epitope insertion to stability and cell-trafficking. ${ }^{20}$ Interestingly, several mutations that interfered with the formation of a correct tertiary structure on the intradiscal surface resulted in mutant opsins that appeared to be retained in the endoplasmic reticulum during heterologous expression, and were complexed with chaperonins. ${ }^{21}$ In general, mutations on the intradiscal surface that might interfere with the formation of a disulfide bond between residues Cys-110 and Cys-187 correlate to a loss of function phenotype. Namely, mutations affecting the ability of these two cysteine residues to juxtapose during translation or membrane translocation affect expression level, transport to the plasma membrane, and the ability of the mutant opsin to regenerate with 11-cis-retinal chromophore to form a proper pigment.

The two conserved cysteine residues on the extracelluar domain, Cys-110 and Cys-187, were shown 
to be essential for proper folding of opsin. ${ }^{22}$ These two residues were shown to form a disulfide linkage in an elegant study in which the four cytoplasmic and three membrane-embedded cysteine residues were removed by site-directed mutagenesis to create a mutant receptor with only the three intradiscal cysteines remaining. ${ }^{23}$ In a related study, the double mutant $\mathrm{C} 110 \mathrm{~A} / \mathrm{C} 187 \mathrm{~A}$ was shown to bind 11-cis-retinal to form a rhodopsin-like pigment. ${ }^{24}$ However, the MII-like photoproduct of the mutant pigment, which could activate transducin in response to light, was considerably less stable than native MII. ${ }^{24}$

Future studies of mutant opsins with defects in folding, membrane insertion, or cell trafficking will be facilitated by the recent report of a methodology to purify regenerated pigment from nonregenerated opsin. The paradigm was developed by using again the opsin mutant containing only the three intradiscal cysteine residues. ${ }^{22}$ It was shown that the nonregenerated opsin, which could not bind 11-cis-retinal, was misfolded and was likely to have an incorrect disulfide bond pairing. ${ }^{25}$ This is additional evidence pointing to the role of the intradiscal domain, and in particular the early formation of a correct disulfide bond linkage, in the proper folding of the opsin apoprotein.

Rhodopsin is also known to be glycosylated on two asparagine residues (Asn-2 and Asn-15) of its aminoterminal tail. A non-glycoslyation rhodopsin, which was prepared in the presence of tunicamycin, was defective in light-dependent activation of transducin. ${ }^{26}$ Site-directed opsin mutants with replacements of these two residues or of neighboring consensus sequence residues were studied as well. It was concluded that glycosylation at Asn-15 was required for full signal transduction activity, but apparently not for correct biosynthesis or folding. ${ }^{26}$

\section{SPECTRAL TUNING AND THE MECHANISM OF THE OPSIN SHIFT}

\section{Spectral Properties of Recombinant Rhodopsins}

The molecular interactions between the opsin protein and the retinal chromophore define the ground state spectral properties of a particular visual pigment. Rhodopsin has been used as a model pigment for a variety of chemical and spectroscopic studies to elucidate the mechanism of the opsin shift. A number of models of the chromophore-binding pocket in rhodopsin have been presented. ${ }^{8.10}$ Relevant to this topic, perhaps the most important single result to arise from the study of mutant bovine rhodopsin pigments was the identification of Glu-113 as the retinylidene Schiff base counterion. ${ }^{27-29}$ This result, combined with the charac- terization of other membrane-embedded carboxylic acid residues by both UV-visible spectroscopy ${ }^{27-30}$ and microprobe resonance Raman ${ }^{31}$ spectroscopy, supported a model with an electrostatically neutral retinal-binding pocket in bovine rhodopsin. ${ }^{8}$ Additional studies including the use of photoaffinity reagents, ${ }^{11,12}$ retinal analogues regenerated with site-directed mutants ${ }^{33}$ or sitedirected mutant pigments ${ }^{30}$ have led to a more complete picture of the amino acid residues in the membraneembedded domain of rhodopsin that interact with the retinal chromophore. Detailed analysis of particular mutant pigments in combination with molecular modeling algorithms should provide a relatively well refined model of the retinal-binding pocket and an understanding of the nature of relevant opsin-chromophore interactions.

\section{Spectral Properties of Recombinant Cone Pigments}

Human trichromatic color vision, at the level of the photoreceptor, requires the presence of three cone pigments with broad overlapping spectral absorption. Three genomic and cDNA clones encoding the opsin apoproteins of these pigments were cloned and characterized. ${ }^{34}$ The amino acid sequences of these opsins are about $41 \%$ identical to that of human rhodopsin. The green and red opsins are about $96 \%$ identical to each other and about $43 \%$ identical to the blue opsin. Previously, the spectral properties of human cone pigments had been studied by a variety of techniques ranging from psychophysical color matching to microspectrophotometry. ${ }^{2}$ Recently, however, the human cone pigment genes were expressed in tissue culture cells, reconstituted with 11-cis-retinal, and studied by UV-visible spectroscopy. ${ }^{35,36}$ The $\lambda_{\max }$ values reported in the two studies were as follows: Blue: $426 \mathrm{~nm}$, green: $530 \mathrm{~nm}$, red: 552 and $557 \mathrm{~nm}$ for polymorphic variants; ${ }^{36}$ and blue: $424 \mathrm{~nm}$, green: $530 \mathrm{~nm}$, and red: $560 \mathrm{~nm} \cdot{ }^{35}$ These studies confirmed the assignments based on genetic analysis of the cloned pigment genes.

Analysis of the arrangement of the cone opsin genes on the X chromosome has led to a detailed understanding of the molecular genetics of inherited variations in color vision. ${ }^{37}$ In males with normal color vision it was proposed that a single red opsin gene resides with one or more green opsin genes in a head-to-tail tandem array. Thus, in one type of color vision defect, anomalous trichromacy, unequal intragenic recombination can theoretically result in an opsin gene that is a hybrid between green and red opsin genes. It was proposed that these hybrids would have anomalous spectral properties. ${ }^{37.38}$ This hypothesis was confirmed experimentally at the level of the photoreceptor pigment by obtaining absorption spectra for heterologously expressed hybrid 
pigments responsible for anomalous trichromacy. ${ }^{39}$ However, recently at the genetic level, quantitative PCR methods have been applied to evaluate in more detail the numbers and ratios of middle- and long-wavelengthabsorbing opsin genes in males with normal color vision. ${ }^{40}$ It was found that many subjects had more numerous opsin genes than previously suggested, and that in many cases more than one long-wavelength opsin gene was present on the $\mathrm{X}$ chromosome. Thus, the exact mechanism of normal and anomalous color vision must be reevaluated..$^{40.41}$

The molecular genetics of blue cone monochromacy has also been investigated. ${ }^{42} \mathrm{~A}$ genetic model to account for the absence of the green and red genes has been tested in transgenic mice..$^{43}$ The results suggest that a conserved $5^{\prime}$-region interacts with the green or red gene promoter to determine which gene is expressed in a given cone cell.

Partial nucleotide sequence information from polymerase chain reaction nucleotide sequencing of monkey visual pigment exons has led to models of spectral tuning and to predictions about the identity of specific amino acids involved in human red/green color vision. ${ }^{44.45}$ New World monkeys, such as marmosets (Callithrix jacchus), tamarins (e.g., Saguinus fuscicollis), and squirrel monkeys (e.g., Saimiri sciureus) have dichromatic vision with a single long-wavelength pigment. However, there is a striking polymorphism in the pigment, such that females with two X-linked opsin genes are effectively trichromatic. Using pairwise comparisons of opsin gene sequences from a number of individual male monkeys with a range of pigment $\lambda_{\max }$ values, a model was proposed in which amino acid residues at three sites account for the spectral variation between human green and red cone pigments: positions 180,277 , and $285 .{ }^{44}$ This model was tested by introducing mutations at these sites in bovine rhodopsin ${ }^{46}$ and by expression of mutant and hybrid human pigment genes. ${ }^{39,47}$ Thus, of the 15 amino acid differences between human green and red pigments, three hydroxylbearing amino acid residues were suggested to be predominantly responsible for the spectral shift: Ser-180, Tyr-277, and Thr-285..$^{39.44,46}$

A more complete analysis of the molecular determinants of red/green color vision was carried out using site-directed mutant pigments and chimeric pigments ${ }^{48.49}$ It was found that of the 15 differences between the 364 amino acid human green and red pigments, seven and only seven amino acid changes were responsible for the observed $31-\mathrm{nm}$ red shift in going from the green to the red pigment: Tyr-116 to Ser, Ala180 to Ser, Thr-230 to Ile, Ser-233 to Ala, Phe-277 to Tyr, Ala-285 to Thr, and Phe-309 to Tyr.
Red and green visual pigments have also been shown to bind chloride ions, resulting in a large red shift in their absorption maxima. An extensive mutagenesis study of 18 different positively charged amino acids identified His-197 and Lys-200, in the extracellular loop linking helices 4 and 5, as the chloride ion binding site in these pigments. ${ }^{49}$ These residues are conserved in long-wavelength-absorbing opsins but not in short-wavelengthabsorbing opsins or rhodopsins, suggesting that the ability to bind chloride was a key event in the evolution of color pigment genes.

\section{Contributions to the Development of Ground-State Structural Models}

The three-dimensional structural model of the lightdriven proton pump, bacteriorhodopsin (BR), ${ }^{50}$ has served as a widely accepted template for the superimposition of the seven putative transmembrane segments of G-protein-coupled receptors. ${ }^{51}$ In particular, it has been inferred that helix segments contiguous in the primary structure are juxtaposed in the tertiary arrangement. In addition, the amino- and carboxyl-termini were expected to be exposed to the extracellular and cytoplasmic domains, respectively, with the helical axes approximately perpendicular to the membrane. These general features appear to be valid according to a recent modeling proposal for G-protein-coupled receptors based on a large number of amino acid sequence comparisons ${ }^{52}$ and the projection structure of bovine rhodopsin at $9-\AA$ resolution determined by cryoelectron microscopy. ${ }^{53}$ However, according to their projection structures, significant differences between the proposed rhodopsin structure and the structure of BR are obvious. ${ }^{53}$ In any case, modeling based on the existing projection map, combined with experimental data on receptor activation including studies of recombinant mutant pigments, allows a tentative assessment of intramolecular processes that may be of functional importance during activation of rhodopsin and of other G-proteincoupled receptors. ${ }^{54}$

\section{LIGHT-INDUCED MOLECULAR CHANGES IN THE MEMBRANE-EMBEDDED DOMAIN}

In rhodopsin, cis to trans photoisomerization ${ }^{55}$ of 11 cis-retinal covalently linked to Lys-296 via a protonated Schiff base (PSB) $)^{56}$ induces the active receptor conformation $\mathrm{R}^{*}$. $\mathrm{R}^{*}$ is spectroscopically defined as metarhodopsin II (MII) absorbing maximally at $380 \mathrm{~nm}$, and $\mathrm{R}^{*}$ activates transducin. ${ }^{57}$ Recent UV-visible, resonance Raman, Fourier-transform infrared (FTIR), and transducin activation experiments on recombinant mutant pigments have led to a better understanding of the photoactivation mechanism of rhodopsin. A critical 
evaluation of the experimentally observed molecular determinants of dark- and light-activated rhodopsin in conjunction with refinement of the hypothetical structural model of rhodopsin should help to design additional mutant rhodopsins to address specific questions regarding the activation mechanism. Key elements of a more detailed discussion of the photoactivation mechanism in rhodopsin ${ }^{58}$ are presented below.

An inactive receptor conformation must be capable of changing to an active conformation which catalyzes nucleotide exchange by a $\mathrm{G}$ protein. In rhodopsin, the 11-cis-retinal chromophore is in its "off" state, but switches to the all-trans geometry, i.e., the "on" state, by photoisomerization. Correspondingly, any amino acid side chain that is involved in such a conformational change must be able to exist in two different sterical and/or electrostatic states, which can be designated as "on" or "off" depending on whether the particular state is observed in the active or inactive receptor, respectively.

Since receptor activation involves conformational changes at different topological locations, an individual amino acid or a structural domain in the chromophorebinding pocket may be in its "on" state without necessarily locking the transducin interaction domain into its active conformation, and vice versa. Thus, a seemingly concerted transition of individual groups may create the overall active conformation, and it is not clear whether or not all observed and measurable structural changes are actually essential for the switching process. A minimal subset of molecular groups may exist that is able to govern the transition to an active receptor conformation irrespective of the binary state ("off"/ "on") of all possible constituents usually participating in receptor activation. Of course, the functional hierarchy of such individual group transitions becomes obvious only in recombinant, or otherwise modified receptors, in which the "on"/"off" state of individual amino acid side chains can be specifically influenced, or even be locked, into one of the two defined states. Ideally, it should be possible to alter the receptor activation pathway such that certain transitions become decoupled from other transitions. It has been argued that the simplified binary model of individual molecular transitions provides a framework to analyze functional data obtained from recombinant rhodopsins..$^{58}$ Experimental results can be related to available structural predictions and may serve as a basis for the design of mutations to specifically address structure-function relations in rhodopsin.

According to this argument, only molecular changes occurring between dark rhodopsin and the $\mathrm{R}^{*}$ state are relevant. Thus, FTIR-difference spectroscopy has proven to be a technique well suited for the study of such light-induced conformational changes in retinal proteins. ${ }^{59}$ FTIR-difference spectroscopy measures frequency shifts of only those vibrational modes which are affected during photoproduct formation, irrespective of whether they are caused by the protein constituents or by the chromophore. In contrast, resonance Raman spectroscopy specifically measures chromophore vibrations and thereby helps to define the chromophore geometry and the Schiff base protonation state without the potential drawback of overlapping absorptions of amino acid side chains. Both techniques have contributed significantly to our knowledge of molecular changes occurring during MII formation in native rhodopsin. Application of these techniques to site-directed rhodopsin mutants have allowed the identification of a number of "on"/"off" states of particular amino acids during $\mathrm{R}^{*}$ formation. Among the putative membrane-embedded carboxylic acid groups, light-induced changes of protonation states or hydrogen-bond strengths were deduced from characteristic frequency shifts of $\mathrm{C}=\mathrm{O}$ stretching vibrations of protonated carboxylic acid groups in FTIR-difference spectra. Their assignment to specific Asp or Glu residues was based on the disappearance of specific difference bands in site-directed mutants and revealed that Asp- $83^{60,61}$ and Glu-122 ${ }^{61}$ are protonated in both dark rhodopsin and MII, whereas Glu-113 is ionized in the dark state and becomes protonated in MII. ${ }^{62}$

Five key individual molecular changes assigned to specific chemical groups in native rhodopsin occur upon MII formation. These light-induced changes occur in the retinal chromophore, at the Schiff base imine, at the counterion Glu-113, and in the hydrogen-bonding environments of Asp-83 and of Glu-122. For example, Glu113 is in its "off" state when it is ionized in dark rhodopsin, where it serves as the counterion to the protonated Schiff base. ${ }^{27-29}$ The "on" state of Glu-113 is created by protonation of its side chain. Based on data derived from mutant pigments, two specific questions can be addressed: (i) which of the "on" states are crucial to maintain the active receptor conformation, and (ii) which of the five known light-induced changes at specific sites in the receptor structure are essential for switching to the active receptor conformation? Answering the first question helps to define the molecular determinants of the active receptor, which need not be identical with the sum of individual "on" states. Answering the second question gives a first approximation of the proposed hierarchy among the contributions of individual molecular states to the energy barrier between the inactive and the active receptor conformation.

\section{Possible Determinants of an Active Receptor Conformation}

Recently, bovine rhodopsin mutants with substitu- 
tions at the site of the Schiff base linkage (Lys-296) and the counterion (Glu-113) were employed to show that a covalent bond to the 11-cis-retinal chromophore is not required for light-dependent activation of transducin. ${ }^{63}$ Furthermore, rhodopsin mutants were reported that have constitutive activity. They activate transducin in the absence of chromophore. ${ }^{17}$ In this context, the main physiological reason for a covalent linkage between opsin and chromophore may be to reduce dark noise and to provide an extremely rapid light-dependent activation. These results emphasize similarities among the opsins and other receptors in terms of the mechanism of G-protein activation.

In several recombinant rhodopsins, the PSB linkage appears to be stabilized by a mutation and yet the photoactivated pigment is still compatible with the $\mathrm{R}^{*}$ state. However, only for the mutant E113A/A117E has this been explicitly shown. ${ }^{64.65}$ The phenotype of this mutant is identical with that of mutant E113A/A117E/ D83N (Fahmy and Sakmar, unpublished results). However, other recombinant pigments likely to form active receptors with a PSB in contrast to the MII state of native rhodopsin include E113Q/A117D, G90D, G90D/ E113Q, A292D, and A292E. Therefore, the Schiff base protonation state does not impose a crucial restriction on the sterical arrangement of the cytoplasmic surface necessary to bind and activate transducin. Interestingly, although a variety of mutations seem to belong to this particular phenotype, they are all characterized by the introduction of a carboxylic acid group at positions usually occupied by a hydrophobic residue. It is likely that this group serves as PSB counterion in the active photoproducts as is suggested by the fact that the double mutant E113Q/G90D forms a blue-shifted photoproduct with a PSB. ${ }^{66}$ The newly introduced electrostatic interaction between a PSB and an "artificial" counterion in the photoproduct does not prevent the active receptor conformation if the charged pair is established between helices three and seven (mutant E113A/A117E) and in another case between helices two and seven (mutant G90D). Obviously, the active conformation of the receptor surface seems to be compatible with a variety of electrostatic Schiff base environments and stabilization of a particular protonation state is not required. Similarly, the hydrogen bonds at protonated Glu-122 and Asp-83, although undergoing bonding energy changes highly characteristic of the $\mathrm{R}^{*}$ formation,${ }^{67}$ cannot be essential to maintain the $\mathrm{R}^{*}$ conformation, since replacement of either residue does not affect transducin activation. ${ }^{61}$ Both groups seem to be well-suited reporter groups for sterical changes at helices two and three without being directly involved in transducin activation or proton transfer reactions.
Correspondingly, "on" states that are crucial for the active receptor conformation have to be looked for among the remaining subset of "on" states common to all photoactivated pigments. Based on the limited number of recombinant pigments studied, candidates for molecular states essential for $\mathrm{R}^{*}$ are the all-trans geometry of the retinal chromophore and a neutral amino acid side chain at position 113 in photoproducts of mutants G90D and A292E. Additional experiments are needed to elucidate whether Glu-113 is neutral, as would be expected if the newly introduced carboxyl groups compete with Glu-113 for electrostatic interaction with the PSB. ${ }^{66}$

\section{Possible Molecular Switches}

Except for photoisomerization of the chromophore, individual "off"/"on" transitions usually occurring in the hydrophobic core of rhodopsin are not required for switching from an inactive to an active conformation. This was shown by the UV photoactivation of the basic form of mutant E113Q, ${ }^{68}$ where neither the charge at residue 113 nor the Schiff base protonation state changed between the dark- and the light-activated state. Both groups were in their respective "on" states already in the dark pigment. Consequently, 11-cis-retinal efficiently abolishes any conformational change that the neutral states of the Schiff base and the Gln-113 side chain may evoke in favor of an active receptor state. This feature has also been demonstrated by the inactivation of the constitutively active mutant opsin E113Q by the addition of 11-cis-retinal, as is the case with other constitutively active mutants which can be regenerated with 11-cis-retinal. ${ }^{69,70}$ The free energy of 11-cis-retinal binding obviously offsets the increase in the free energy of the mutant opsin due to incorporation of a neutral amino acid at position 113. Therefore, a sufficiently high energy barrier between the dark and photoactivated state of the 11-cis-retinal regenerated rhodopsin is reestablished which prevents measurable dark activity. It is this mutant which also demonstrates that in a given 11-cis-retinal regenerated opsin, photoactivation may or may not involve a change of the Schiff base protonation state. Depending on $\mathrm{pH}$, a protonated or an unprotonated 11-cis-retinal Schiff base photoisomerizes to the unprotonated all-trans-retinal chromophore and forms an active-state $\mathrm{R}^{*}{ }^{68}$ Since the opsin is the same in both cases it is reasonable that the active states thus created are identical. This mitigates against an essential involvement of the Schiff base protonation state in the switching mechanism, which is also in agreement with the existence of an active receptor conformation with a PSB as shown for mutant pigment E113A/A117E. It is an astonishing result that a variety of electrostatic transi- 
tions occurring in the retinal-binding sites in various recombinant rhodopsins are compatible with the lightdependent formation of a cytoplasmic receptor surface that is recognized by transducin and catalyzes nucleotide exchange.

The different electrostatic chromophore environments obviously allow spectral tuning of the photoreceptor without interfering with the photoactivation mechanism itself. Most surprisingly, molecular substates typical of the inactive receptor can be mutationally stabilized in the hydrophobic core and yet coexist with an active cytoplasmic surface conformation. This has not been anticipated on the basis of biochemical modification of native rhodopsin, showing that Schiff base deprotonation is required for the formation of MII. ${ }^{71}$ Similarly, a model which elegantly unifies the possible causes of constitutive activity in a large number of recombinant opsins suggests breakage of a salt bridge between Glu-113 and Lys-296 as a key event in receptor activation. ${ }^{17,69,70}$ Do additional recent mutational data argue against the importance of an electrostatic switching mechanism in the hydrophobic core of rhodopsin or other G-protein-coupled receptors?

Site-directed mutagenesis has allowed otherwise necessary electrostatic transitions in the hydrophobic domain of rhodopsin to be modified or even abolished. Only 4 of the 16 theoretically possible transitions among 4 combinations of Schiff base and Glu-113 protonation states in the dark and photoactivated pigments have been mimicked by recombinant pigments. To date, site-directed mutagenesis has not created a pigment in which the "off" state of Glu-113 is stabilized in the photoproduct; i.e., no pigments have been described in which Glu-113 is known to be ionized before and after photoisomerization of the retinal chromophore. In perfect analogy to the mutant E113A/A117E, in which Schiff base deprotonation is decoupled from receptor activation, this hypothetical recombinant pigment would answer the question of whether Glu-113 protonation is indeed essential. The available data favor the necessity of a neutral side chain of Glu-113 in the active conformation. This is primarily based on two results; Glu-113 is the Schiff base proton acceptor in MII, ${ }^{61.62}$ and Schiff base deprotonation is required for $\mathrm{R}^{*}$ formation..$^{71}$ In addition, mutational incorporation of a neutral side chain at position 113 causes receptor activity in the absence of chromophore. ${ }^{69,70}$ This, of course, does not imply that Glu-113 has to be ionized in the inactive receptor, as shown by the lack of dark activity of 11-cis-retinal regenerated mutants.

However, if Glu-113 is ionized in a dark pigment, as is the case with native rhodopsin, then an electrostatic switch has to exist which neutralizes Glu-113 in order to create a putatively essential determinant of the active receptor conformation. Therefore, it is likely that in native rhodopsin, an electrostatic switch at Glu-113 exists in addition to the sterical switch provided by photoisomerization. Both are coupled via proton transfer from the Schiff base to Glu-113. Mechanistically, however, the "on" state of Glu-113 can be anticipated in the inactive receptor without causing dark activity, due to 11-cis-retinal being a strong antagonist. This opens the possibility that naturally occurring UV-absorbing pigments with a neutral amino acid at the position homologous to 113 in rhodopsin may use the same molecular activation mechanism as do rhodopsins containing a protonated retinal Schiff base chromophore. The UV-absorbing pigments may use the sterical switch alone by anticipating the presumably essential "on" state of the amino acid at 113 position already in the dark state. The fact that insect UV-absorbing pigments form photoproducts with PSB chromophores does not indicate an activation mechanism different from that in rhodopsin. The results with mutants E113A/A117 $\mathrm{E}^{64,65}$ and $\mathrm{E} 113 \mathrm{Q}^{68}$ show that the Schiff base protonation state per se is an essential determinant neither of the active nor of the inactive receptor. Therefore, it does not have to be part of a molecular switching mechanism. However, Schiff base deprotonation must participate in receptor activation in native rhodopsin for the reasons explained above.

\section{Other Moieties Involved in Regulation of Receptor Activity}

Additional amino acids have been identified in the hydrophobic core of rhodopsin which affect the absorption properties, the photoreaction, and the transducin activation efficiency of rhodopsin. ${ }^{72}$ But only for some of them have physical or chemical alterations of their functional groups been suggested to occur upon MII formation. His-211, although not generally required for MII formation, reduces the amount of MII formed in digitonin-membrane micelles, and it has been suggested that protonation of His-211 may explain the $\mathrm{pH}$-dependency of the MI-MII equilibrium ${ }^{73}$ described for rhodopsin in disc membranes..$^{74}$ In a functional assay, however, the H211F mutant was found to activate transducin with an efficiency similar to that of native rhodopsin, ${ }^{69}$ which was not expected on the basis of an MI-like photoproduct absorption spectrum. However, the compatibility of an active receptor conformation with "off" states of particular molecular groups, renders the UV-spectroscopic characterization of the rhodopsin states ambiguous. Although mutant pigment photoproducts of the pigments may exhibit MI-like absorption spectra, an MII-like conformation may coexist as shown 
for E113A/A117E. Therefore, further experiments which allow more direct assessment of the protein conformation are needed to elucidate the impact of His-211 on receptor conformation. In a recent FTIR study, cysteines have been suggested to be implicated in the photoactivation mechanism as well, but site-specific band assignment is not yet available. ${ }^{75}$

\section{LIGHT-INDUCED MOLECULAR CHANGES ON THE CYTOPLASMIC SURFACE}

Synthetic rhodopsin-derived peptides have been shown to compete with native rhodopsin for transducin binding. ${ }^{76}$ This has allowed the identification of the cytoplasmic loops connecting helix three with helix four and helix five with helix six, as well as a putative loop between the cytoplasmic termination of helix seven and the palmitoylated Cys-322 and Cys- 323 residues, as transducin-binding sites. Site-directed mutagenesis has further characterized groups of amino acids in these regions implicated in transducin binding and activation. ${ }^{77,78}$ However, light-dependent alterations of their physical or chemical states are not well characterized, rendering a distinction of "on" and "off" states of individual amino acid side chains difficult, even if the importance of specific amino acids (e.g., the charged pair Glu-134/Arg-135) ${ }^{79}$ for transducin activation is established. Infrared spectroscopic determination of protonation states or hydrogen bonding of specific amino acid side chains on the cytoplasmic surface of rhodopsin is, to date, less advanced than for the hydrophobic core of the receptor. This is due in part to the relatively small contribution of water-exposed amino acids to infrared absorption changes in typical FTIR samples (hydrated films) ${ }^{80}$

Other techniques have been more successful in monitoring light-dependent structural alterations occurring during MIl formation. It has been shown by time-resolved $^{81}$ and static electron paramagnetic resonance (EPR) spectroscopy studies ${ }^{82}$ on site-specific spin-labeled rhodopsin that the cytoplasmic terminations of helices three and seven undergo structural rearrangements in the vicinities of Cys-140 and Cys-316, respectively. These changes have been specifically assigned to the MII conformation. Cys-140 is close to the highly conserved Glu or Asp/Arg/Tyr triad at the cytoplasmic border of helix three (positions 134-136 in rhodopsin), which has attracted attention in earlier studies because of its possible general importance for the function of Gprotein-coupled receptors. It has been shown that replacement of Glu- 134 by Gln renders the photoactivated pigment about 8 -fold more efficient in activating transducin at alkaline $\mathrm{pH}$ than recombinant native rhodopsin..$^{79}$ Therefore, it has been suggested that Glu134 is a good candidate for regulation of the transducinbinding region and may undergo a light-induced transition from an ionized to a protonated state. ${ }^{70.79}$ Recent measurements of light-induced $\mathrm{pH}$ changes in the bulkwater phase monitored simultaneously with $\mathrm{R} *$ formation of the mutants E134Q and E134D showed the involvement of Glu- $134^{83}$ in proton uptake reactions. ${ }^{84}$

According to these results, it is likely that Glu-134 itself is a group which becomes protonated in MII. However, FTIR difference spectroscopy to date has failed to detect any $\mathrm{C}=\mathrm{O}$ stretching vibration assignable to the putative protonation of Glu-134 in detergent micelles (Fahmy and Sakmar, unpublished results) or in reconstituted membranes. ${ }^{60}$

The structural change detected by EPR spectroscopy may be directly related to protonation of Glu-134, which is expected to significantly alter the hydrogen-bonding properties of this amino acid. A rearrangement of neighboring hydrogen-bonding partners may then explain the conformational change. There is no straightforward approach to identify surface groups which interact with Glu-134, although the $\mathrm{pH}$-profile of transducin activation, ${ }^{79}$ as well as the abolishment of the uptake of two protons in E134Q, ${ }^{83}$ suggests the existence of other titratable groups influenced by Glu-134. Since Glu-134 is at the cytoplasmic border of helix three in a region demonstrated to undergo structural alterations upon MII formation, it is not clear from the helical arrangement model which amino acids may interact with Glu- 134 . Interestingly, His-65 and, to a lesser extent, His-152 have been shown to affect the receptor conformation since their replacement favors MII formation, as measured by $380-\mathrm{nm}$ absorbance, ${ }^{73}$ and this phenotype is similar to the one reported for Glu-134 mutations. ${ }^{85}$

\section{MECHANISTIC COUPLING OF LIGHT-INDUCED CONFORMATIONAL CHANGES TO TRANSDUCIN ACTIVATION}

As mentioned above, the domains of rhodopsin that interact with transducin have been studied by site-directed mutagenesis of bovine rhodopsin. Relatively large segments of cytoplasmic loops are required for proper rhodopsin-transducin interaction. ${ }^{86}$ However, single amino acid substitutions within these domains can have dramatic effects on transducin activation. ${ }^{27.30}$ In addition, transducin binding and activation of bound transducin were shown to be discrete steps involving different surface domains of the receptor. ${ }^{77}$ Mutant pigments with alterations of the second and third cytoplasmic loops were characterized that formed spectrally normal MII-like photoproducts that bound transducin. ${ }^{77}$ 
However, the bound transducin was apparently defective in the release of GDP, which accounts for the block in pigment-catalyzed GTP uptake or GTPase activity by transducin observed when the mutant pigments were assayed.$^{87}$

One striking result of recent spectroscopic and functional studies on recombinant rhodopsins is the mutationally mimicked coexistence of "on" and "off" states of specific amino acid side chains for which light-dependent chemical or physical transitions have been characterized in native rhodopsin. It has been discussed above that a PSB is compatible with an active receptor in mutant E113A/A117E, although transducin activation is reduced by about $30 \%$. Obviously, other "on" states efficiently determine the active conformation, rendering the Schiff base protonation state a weak restriction for the conformation of the transducin interaction domain on the cytoplasmic surface. In analogy, mutational stabilization of an "on" state may, under certain conditions, enhance transducin activation, as has been demonstrated for the photoproduct of E134Q. ${ }^{79}$ In agreement with this notion of a weighted additivity of molecular substates, mimicking the "on" state of Glu134 by substituting Gln in a given mutational background is expected to raise transducin activation in the particular system. This has indeed been observed for the pair of constitutively active mutants $\mathrm{K} 296 \mathrm{H}$ and $\mathrm{K} 296 \mathrm{H} / \mathrm{E} 134 \mathrm{Q} .^{70}$ Even in the absence of retinal, the cytoplasmic surface seems to maintain at least a partial structural integrity in the sense that mutations within the transducin-binding domain change the phenotype in the same way as in the light-activated recombinant pigment. This agrees with a previously suggested partial conformational independence between transitions occurring in the hydrophobic core (photoisomerization and proton transfer to Glu-113) and on the cytoplasmic surface (proton uptake, probably by Glu-134 and an as yet unidentified group) ${ }^{83}$ An exciting question is whether these observations can be exploited to create physically or mutationally even more severe mismatches between "on" and "off" states.

Due to the free energy of transducin binding to MII, at least some of the "on" states produced by pigment activation can be stabilized by transducin binding. This constitutes the physical counterpart to the more specific and efficient mutational blockade of "on" states. An interesting experiment was carried out by reisomerizing the all-trans chromophore in transducin-bound MII in order to create a mismatch between the isomeric "off" state 11-cis-retinal and a partially stabilized MII conformation. ${ }^{84}$ This approach revealed that transducin stays bound to the cytoplasmic surface of $\mathrm{R}^{*}$ even if all-transretinal is replaced by 11-cis-retinal by photoconversion.
Although the all-trans-chromophore and a neutral residue at position 113 may be essential determinants of an active receptor conformation, this photoconversion experiment shows that there is no tight sterical coupling between the transducin binding loops and the all-transchromophore geometry once transducin has bound. This agrees well with the existence of a large number of constitutively active mutant opsins carrying amino acids of variable sizes at position $296 .{ }^{70}$ It should be emphasized, however, that this result does not at all contradict the notion of photoisomerization being a necessary switch to reach the conformation of the free receptor which allows transducin binding. Only the latter step provides additional energy which introduces intermolecular interactions that may reorganize the hierarchy of intramolecular "on"/"off" transitions.

The proposition that amino acid replacements as well as the described photoconversion create hybrid structures of activity-promoting and activity-inhibiting substates rather than mimicking a well-defined conformation of the native receptor (e.g., the conformation of a spectroscopically defined photointermediate of rhodopsin) is paralleled by a recent study of constitutively active opsin mutants. Although Lys-296 replacements allow transducin binding and activation in the absence of chromophore, the corresponding "active receptor conformation" is not necessarily recognized by rhodopsin kinase ${ }^{88}$ This supports the importance of specific sterical interactions of all-trans-retinal with the protein environment in native MII.

The light-dependent alterations of the protonation or hydrogen-bonding states of specific molecular groups in the hydrophobic core and on the cytoplasmic surface of rhodopsin, when combined with known structural information, allow several conclusions regarding possible activation mechanisms in rhodopsin. Structural data include the proposed helix arrangement ${ }^{52.54}$ as well as FTIR results obtained with retinal analogues allowing identification of sterically important regions of the chromophore. A characteristic feature of the projection structure of rhodopsin is the smaller distance between the axes of helices three and five as compared to the distance in BR, where these helices are effectively separated by helix four. The rhodopsin structure accommodating the 11-cis isomer seems to have a more compact arrangement of helices three, four, and five than the more elongated projection structure of $\mathrm{BR}$, in which an all-trans isomer is incorporated. In a first approximation, photoisomerization in rhodopsin may be expected to cause chromophore-protein interactions which introduce sterical perturbations near the three/four and/or the four/five helical interface(s). Although this notion agrees with an earlier suggestion of relative movements 
among helices three to five ${ }^{82}$ or three to $\operatorname{six}^{89}$ it does not necessarily imply global movements of helices. It may be that at these helical interfaces, amino acid side chains may be expected to experience changes in van der Waal contacts or hydrogen-bond strengths upon photoisomerization of the retinal chromophore.

A possible functional importance of the relative positions of helices three and four of rhodopsin is also suggested on the basis of a conserved disulfide bond between these helices on the extracellular receptor domain, ${ }^{19}{ }^{22.23}$ which stabilizes the MII conformation. ${ }^{24}$ In terms of the chromophore, the suggested localization of light-induced sterical changes would be expected to affect mostly locations distal to the Schiff base linkage, which is situated at the interfaces of helices two, three, and seven. ${ }^{52.54 .66}$

Such a partitioning of crucial protein-chromophore interactions along the retinal is supported by FTIR studies on rhodopsin regenerated with retinal analogues showing that the sterical impacts of the two methyl groups of retinal are not equivalent. The more distal 9methyl group is essential to induce those conformational changes which give rise to the typical MII absorption changes. ${ }^{90} \mathrm{~A}$ drastic reduction in transducin activation is observed in 9-desmethyl retinal rhodopsin. In contrast, the 13-methyl group which is closer to the Schiff base linkage does not play a crucial role to sustain the MII conformation..$^{91}$

Further evidence for the importance of sterical interactions distal from the Schiff base comes from FTIR studies using ring-modified retinal analogues. Increased flexibility, as in 5,6-dihydro ${ }^{92}$ or 7,8-dihydro analogues ${ }^{93}$ reduces the usually observed torsions along the retinal chain in the batho intermediates at $80 \mathrm{~K}$. In addition, the protein conformational changes observed at temperatures which stabilize the MI or MII intermediates differ from those observed in native rhodopsin. In an extreme case, illumination of a pigment regenerated with a retinal analogue lacking the $\beta$-ionone ring fails to induce the complete set of infrared absorption changes typical of the MII conformation and results in reduced transducin activation. ${ }^{94}$ Therefore, the $\beta$-ionone ring must transmit important sterical changes to the protein. According to the proposed model of light-induced sterical perturbation at the helix three/four interface and the proposed anchoring of the $\beta$-ionone ring near helix four, one would expect some effect of ring modifications on infrared absorption changes from amino acids at the helix three/four interface. The model proposed by Baldwin ${ }^{52.54}$ locates Glu-122 at the helix three/four interface. The infrared absorption change assigned to the reduction of hydrogen bonding of Glu-122 upon MII formation was indeed not observed in rhodopsin regen- erated with a retinal analogue which lacks the $\beta$-ionone ring, whereas Asp-83 showed a normal absorption change. The proposed vicinity of the $\beta$-ionone ring and Glu-122 is also in agreement with resonance Raman data on mutant pigment E122Q ${ }^{31}$ Interestingly, infrared absorption changes of Glu-122 occur prior (in MI) to those of Asp-83 (in MII). This is even more pronounced in mutant E122D, which exhibits hydrogen-bond alterations of Asp-122 already in the batho intermediate at $80 \mathrm{~K}$, when most of the protein conformation is thermally fixed. This suggests even tighter sterical coupling of Asp-122 to the primary photochemical event than is the case for Glu-122. ${ }^{95}$

Taken together, it seems likely that the primary sterical effect of photoisomerization is mainly localized at the helix three/four interface where it is sensed by Glu-122. Ensuing thermal relaxation would transmit the sterical alteration to the cytoplasmic surface and may thus explain why a structural change upon MII formation is observed particularly in the loop connecting helix three and four. ${ }^{81.82}$

\section{CONCLUSIONS}

Over the last several years, a remarkable amount of information about structure-function relationships in Gprotein-coupled receptors has been obtained using techniques of molecular biology. In the study of visual pigments in particular, site-directed mutant pigments have been employed to elucidate key structural elements, the opsin-shift mechanism, and the mechanism of receptor photoactivation. For example, the combination of wellestablished spectroscopic methods with site-directed mutagenesis has revealed an unexpected variety of active receptor states created by photoisomerization of 11cis-retinal regenerated mutant opsins. In particular, hybrid states in which features of the active receptor conformation are anticipated in the inactive state have been described. The efficiency of transducin activation seems to be roughly determined by a weighted sum of individual molecular substates which promote or inhibit activity. Among these, retinal isomerization contributes through sterical interactions to overcome the energy barrier between the dark- and photoactivated-pigment conformation.

In addition, the protonation changes of Glu-113 (and most likely Glu-134) are expected to determine the amount of electrostatic energy contributions. A specific interaction of the $\beta$-ionone ring and the 9 -methyl group of all-trans-retinal with amino acids at the helix three/ four interface seems to be important to induce proton transfer from the all-trans PSB to Glu-113 as a prerequisite for transducin activation. However, the decoupling of electrostatic changes in the retinal-binding pocket 
from the photoactivation process in a number of recombinant pigments calls for further experimentation to elucidate the detailed role of Glu-113 protonation for activation of rhodopsin.

\section{REFERENCES AND NOTES}

(1) (a) Wald, G. Nature 1968, 219: 800. (b) Chabre, M. Annu. Rev. Biophys. Chem. 1985, 14: 331. (c) Stryer, L. J. Biol. Chem. 1991, 266: 10711.

(2) Bowmaker, J.K.; Dartnall, H.J.A. J. Physiol. 1980, 298: 501.

(3) (a) Applebury, M.L.; Hargrave, P.A. Vision Res. 1986, 26: 1881. (b) Yokoyama, R.; Knox, B.E.; Yokoyama, S. Invest. Ophth. Vis. Sci. 1995, 36: 939. (c) Johnson, R.L.; Grant, K.B.; Zanke, T.C.; Boehm. M.F.; Merbs, S.L.; Nathans, J.; Nakanishi. K. Biochemistry 1993, 32: 208.

(4) Benovic, J.L.; Mayor, F.J., Somers, R.L.; Caron, M.G.; Lefkowitz, R.J. Nature 1986, 321: 869.

(5) (a) Ovchinnikov, Y.A. FEBS Lett. 1982, 148: 179. (b) Hargrave, P.A.; McDowell, J.H.: Curtis, D.R.; Wang. J.K.; Jusczak, E.; Fong, S.L.; Mohanna Rao, J.K.; Argos, P. Biophys. Struct. Mech. 1983, 9: 235.

(6) (a) Nathans, J.; Hogness, D.S. Cell 1983, 34: 807. (b) Nathans, J.; Hogness, D.S. Proc. Natl. Acad. Sci., USA 1984, 81: 4851.

(7) Dixon, R.A.F.; Kobilka, B.K.; Strader, D.J.; Benovic, J.L.; Kohlman, H.G.; Frielle, T.; Bolanowski, M.A.: Bennett, C.D.; Rands, E.; Diehle, R.E.; Mumford, R.A.; Slater, E.E.; Sigal, I.S.; Caron, M.G.; Lefkowitz, R.J.; Strader, C.D. Nature 1986, 321: 75.

(8) Birge, R.R. Biochim. Biophys. Acta 1990, 1016: 293.

(9) Siebert, F. Methods Enzymol. 1990. 189: 123.

(10) Honig. B.; Dinur, U.; Nakanishi, K.; Balogh-Nair, V.; Gawinowica, M.A.; Arnaboldi, M.; Motto, M.G. J. Am. Chem. Soc. 1980, 101: 7084.

(11) Nakayama, T.A.; Khorana, H.G. J. Biol. Chem. 1990, 265: 15762.

(12) Zhang, H.; Lerro, K.A.; Yamamoto, T.; Lien, T.H.; Sastry, L.; Gawinowicz, M.A.; Nakanishi, K. J. Am. Chem. Soc. 1994, 116: 10165.

(13) (a) Khorana, H.G. J. Biol. Chem. 1992, 267: 1. (b) Nathans, J. Biochemistry 1992, 31: 4923.

(14) Dryja, T.P.; McGee, T.L.; Reichel, E.; Hahn, L.B.; Cowley, G.S.; Yandell, D.W.; Sandberg, M.A.; Berson, E.L. Nature 1990, 343: 364

(15) (a) Dryja, T.P.; Hahn, L.B.; Cowley, G.S.; McGee, T.L.; Berson, E.L. Proc. Natl. Acad. Sci., USA 199188 : 9370. (b) Sung, C.-H.; Davenport, C.M.; Hennessey, J.C.; Maumenee, I.H.; Jacobson, S.G.; Heckenlively, J.R.; Howakowski, R.; Fishman, G.; Gouras, P.; Nathans, J. Proc. Natl. Acad. Sci., USA 1991, 88: 6481.

(16) (a) Kaushal, S.; Khorana, H.G. Biochemistry 1994, 33: 6121. (b) Sung, C.-H.; Schneider, B.G.; Agarwal, N.; Papermaster, D.S.; Nathans, J. Proc. Natl. Acad. Sci., USA 1991, 88: 8840.

(17) Robinson, P.R.; Cohen, G.B.; Zhukovsky, E.A.; Oprian, D.D. Neuron 1992, 9: 719.

(18) (a) Olsson, J.E.; Gordon, J.W.; Pawlyk, B.S.; Roof, D.; Hayes, A.; Molday, R.S.; Mukai, S.; Cowley, G.S.;
Berson, E.L.; Dryja, T.P. Neuron 1992, 9: 815. (b) Roof, D.J.; Adamian, M.; Hayes, A. Invest. Ophth. Vis. Sci. 1994, 35: 4049.

(19) Doi, T.; Molday, R.S.; Khorana, H.G. Proc. Natl. Acad. Sci., USA 1990, 87: 4991.

(20) Borjigin, J.; Nathans, J. J. Biol. Chem. 1994, 269: 14715.

(21) Anukanth, A.; Khorana, H.G. J. Biol. Chem. 1994, 269 : 19738.

(22) Karnik, S.S.; Sakmar, T.P.; Chen, H.-B.; Khorana, H.G. Proc. Natl. Acad. Sci., USA 1988, 85: 8459.

(23) Karnik, S.S.; Khorana, H.G. J. Biol. Chem. 1990, 265: 17520.

(24) Davidson, F.F.; Loewen, P.C.; Khorana, H.G. Proc. Natl. Acad. Sci., USA 1994, 91: 4029.

(25) Ridge, K.D.; Lu, Z.; Liu, X.; Khorana, H.G. Biochemistry 1995, 34: 3261 .

(26) Kaushal, S.; Ridge, K.D.; Khorana, H.G. Proc. Natl. Acad. Sci., USA 1994, 91: 4024.

(27) Sakmar, T.P.; Franke, R.R.; Khorana, H.G. Proc. Natl. Acad. Sci., USA 1989, 86: 8309.

(28) Zhukovsky, E.A.; Oprian, D.D. Science 1989, 246: 928.

(29) Nathans, J. Biochemistry 1990, 29: 937.

(30) Nakayama, T.A.; Khorana, H.G. J. Biol. Chem. 1991, 266: 4269 .

(31) Lin, S.W.; Sakmar, T.P.; Franke, R.R.; Khorana, H.G.; Mathies, R.A. Biochemistry 1992, 31: 5105.

(32) Bhattacharya, S.; Ridge, K.D.; Knox, B.E.; Khorana, H.G. J. Biol. Chem. 1992, 267: 6763.

(33) Ridge, K.D.; Bhattacharya, S.; Nakayama, T.A.; Khorana, H.G. J. Biol. Chem. 1992, 267: 6770.

(34) Nathans, J.; Thomas, D.; Hogness, D.S. Science 1986, 232: 193.

(35) Oprian, D.D.; Asenjo, A.B.; Pelletier, S.L. Biochemistry 1991, 30: 11367.

(36) Merbs, S.L.; Nathans, J. Nature 1992, 356: 433.

(37) Nathans, J.; Piantanida, T.P.; Eddy, R.L.; Shows, T.B.; Hogness, D.S. Science 1986, 232: 203.

(38) Neitz, J.; Neitz, M.; Jacobs, G.H. Nature 1989, 342: 679.

(39) Merbs, S.L.; Nathans, J. Science 1992, 258: 464.

(40) Neitz, M.; Neitz, J. Science 1995, 267: 1013.

(41) Neitz, J.; Neitz, M. In Molecular Genetics of Inherited Eye Disorders; Wright, A.F.; Jay, B., Eds.; Harwood Academic Publishers: Chur, Switzerland, 1994, p. 217.

(42) Nathans, J.; Davenport, C.M.; Maumenee, I.H.; Lewis, R.A.; Hejtmancik, J.F.; Litt, M.; Lovrien, E.; Weleber, R.; Bachynski, B.; Zwas, F.; Klingaman, R.; Fishman, G. Science 1989, 245: 831.

(43) Wang, Y.; Macke, J.P.; Merbs, S.L.; Zack, D.J.; Klaunberg, B.; Bennet, J.; Gearhart, J.; Nathans, J. Neuron 1992, 9: 429.

(44) Neitz, M.; Neitz, J.; Jacobs, G.H. Science 1991, 252: 971.

(45) Williams, A.J.; Hunt, D.M.; Bowmaker, J.K.; Mollon, J.D. EMBO J. 1992, 11: 2039.

(46) Chan, T.; Lee, M.; Sakmar, T.P. J. Biol. Chem. 1992, 267: 9478.

(47) Merbs, S.L.; Nathans, J. Photochem. Photobiol. 1993, 58: 705 . 
(48) Asenjo, A.B.; Rim, J.; Oprian, D.D. Neuron 1994, 12: 1131.

(49) Wang, Z.; Asenjo, A.B.; Oprian, D.D. Biochemistry 1993, 32: 2125.

(50) Henderson, R.; Baldwin, J.M.; Ceska, T.A.; Zemlin, F.; Beckmann, E.; Downing, K.H. J. Mol. Biol. 1990, 213: 899.

(51) Röper, D.; Jacoby, E.; Krüger, P.; Engels, M.; Grötzinger, J.; Wollmer, A; Strassburger, W. J. Recep. Res. 1994, 14: 167.

(52) Baldwin, J.M. EMBO J. 1993, 12: 1693.

(53) Schertler, G.F.X.; Villa, C.; Henderson, R. Nature 1993, 362: 770.

(54) Baldwin, J.M. Curr. Opin. Cell Biol. 1994, 6: 180.

(55) (a) Yoshizawa, T.; Wald, G. Nature 1963, 197: 1279. (b) Bownds, D. Nature 1967, 216: 1178. (c) Dratz, E.A.; Hargrave, P.A. Trends Biochem. Sci. 1983, 8: 128.

(56) Oseroff, A.R.; Callender, R.H. Biochemistry 1974, 13: 4243.

(57) (a) Kibelbek, J; Mitchell, D.C.; Beach, J.M.; Litman, B.L. Biochemistry 1991, 30: 6761. (b) Hofmann, K.P. Photobiochem. Photobiophys. 1986, 13: 309. (c) Lewis, J.L.; Kliger, D.S. J. Bioenerg. Biomembr. 1992, 24: 201. (d) Stryer, L. Annu. Rev. Neurosci. 1986, 9: 87.

(58) Fahmy, K.; Siebert, F.; Sakmar, T.P. Biophys. Chem. 1995, 56: 171.

(59) Rothschild, K.J. J. Bioenerg. Biomembr. 1992, 24: 147.

(60) Rath, P.; DeCaluwé, L.L.J.; Bovee-Geurts, P.H.M.; de Grip, W.J.; Rothschild, K.J. Biochemistry 1993, 32: 10276.

(61) Fahmy, K.; Jäger, F.; Beck, M.; Zvyaga, T.A.; Sakmar, T.P.; Siebert, F. Proc. Natl. Acad. Sci., USA 1993, 90: 10206.

(62) Jäger, F.; Fahmy, K.; Sakmar, T.P.; Siebert, F. Biochemistry 1994, 33: 10878.

(63) Zhukovsky, E.A.; Robinson, P.R.; Oprian, D.D. Science 1991, 251: 558.

(64) Zvyaga, T.A.; Fahmy, K.; Sakmar, T.P. Biochemistry 1994, 33: 9753.

(65) Fahmy, K.; Siebert, F.; Sakmar, T.P. Biochemistry 1994, 33: 13700.

(66) Rao, V.R.; Cohen; G.B.; Oprian, D.D. Nature 1994, 367: 639 .

(67) Klinger, A.L.; Braiman, M.S. Biophys. J. 1992, 63: 1244.

(68) Fahmy, K.; Sakmar, T.P. Biochemistry 1993, 32: 9165.

(69) Cohen, G.B.; Oprian, D.D.; Robinson, P.R. Biochemistry 1992, 31: 12592.

(70) Cohen, G.B.; Yang, T.; Robinson, P.R.; Oprian, D.D. Biochemistry 1993, 32: 6111.

(71) (a) Longstaff, C.; Calhoon, R.D.; Rando, R.R. Proc. Natl. Acad. Sci., USA 1986, 83: 4209. (b) Ganter, U.M.; Longstaff, C.; Pajares, M.A.; Rando, R.R.; Siebert, F. Biophys. J. 1991, 59: 640.
(72) Oprian, D.D. Curr. Opin. Neurobiol. 1992, 2: 428.

(73) Weitz; C.J.; Nathans, J. Neuron 1992, 8: 465.

(74) (a) Mathews, R.G.; Hubbard, R.; Brown, P.K.; Wald, G. J. Gen. Physiol. 1963, 47: 215. (b) Bennet, N. Biochem. Biophys. Res. Commun. 1978, 83: 457. (c) Bennet, N. Eur. J. Biochem. 1980, 111: 99. (d) Parks, J.H.; Liebman, P.A. Biochemistry 1984, 23 : 5054.

(75) Rath, P.; Bovee-Geurts, P.H.M.; de Grip; W.J.; Rothschild, K.J. Biophys. J. 1994, 66: 2085.

(76) König, B.; Arendt, A.; McDowell, J.H.; Kahlert, M.; Hargrave; P.A.; Hofmann, K.P. Proc. Natl. Acad. Sci., USA 1989, 86: 6878.

(77) Franke, R.R.; König, B.; Sakmar, T.P.; Khorana, H.G.; Hofmann, K.P. Science 1990, 250: 123.

(78) Franke, R.R.; Sakmar, T.P.; Graham, R.M.; Khorana, H.G. J. Biol. Chem. 1992, 267: 14767.

(79) Fahmy, K.; Sakmar, T.P. Biochemistry 1993, 32: 7229.

(80) Ganter, U.M.; Charitopoulos, T.; Virmaux, N.; Siebert, F. Photochem. Photobiol. 1992, 56: 57.

(81) Farahbakhsh, Z.T.; Hideg, K.; Hubbell, W.L. Science 1993, 262: 1416.

(82) Resek, J.F.; Farahbakhsh, Z.T.; Hubbell, W.L.; Khorana, H.G. Biochemistry 1993, 32: 12025.

(83) Arnis, S.; Fahmy, K.; Hofmann, K.P.; Sakmar, T.P. J. Biol. Chem. 1994, 269: 23879.

(84) Arnis, S.; Hofmann, K.P. Proc. Natl. Acad. Sci., USA 1993, 90: 7849.

(85) Weitz, C.J.; Nathans, J. Biochemistry 1993, 32: 14176.

(86) Franke, R.R.; Sakmar, T.P.; Graham, R.M.; Khorana, H.G. J. Biol. Chem. 1992, 267: 14767.

(87) Ernst, O.; Hofmann, K.P.; Sakmar, T.P. J. Biol. Chem. 1995, 270: 10580.

(88) Robinson, P.R.; Buczylko, J.; Oghuru, H.; Palczewski, K. Proc. Natl. Acad. Sci., USA 1994, 91: 5411.

(89) (a) Pellicone, C.; Cook, N.J.; Nullans, G.; Virmaux, N. FEBS 1985, 181: 184. (b) Pellicone, C.; Nullans, G.; Cook, N.J.; Virmaux, N. Biochem. Biophys. Res. Commun. 1985, 127: 816.

(90) Ganter, U.M.; Schmid, E.D.; Perez-Sala, D.; Rando, R.R.; Siebert, F. Biochemistry 1989, 28: 5954.

(91) Ganter, U.M.; Gärtner, W.; Siebert, F. Eur. Biophys. J. 1990, 18: 295.

(92) Ganter, U.M.; Kashima, T.; Sheves, M.; Siebert, F. J. Am. Chem. Soc. 1991, 113: 4087.

(93) Palings, I.; Pardoen, J.A.; van den Berg, E.; Winkel, C.; Lugtenburg, J.; Mathies, R.A. Biochemistry 1987, $26: 2544$.

(94) Jäger, F.; Jäger, S.; Kräutle, O.; Friedman, N.; Sheves, M.; Hofmann, K.P.; Siebert, F. Biochemistry 1994, 33: 7389.

(95) Jäger, F.; Sakmar, T.P.; Siebert, F.; In Fifth International Conference on the Spectroscopy of Biological Molecules; Theophanides, T.; Anatassopoulou, J.; Fotopoulos, N., Eds.; Kluwer Academic Publishers: Dordrecht, 1993, p. 223. 